February - 2018

\title{
Editorial - Volume 19, Issue 1
}

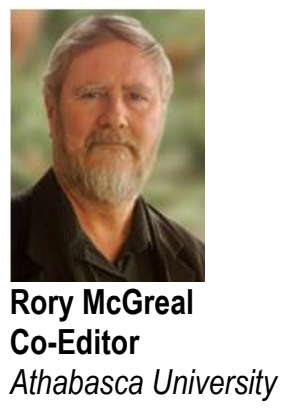

Welcome to this first edition of IRRODL for 2018. This issue begins with several articles focusing on learners, including papers on collaborative learning, self-pacing, interaction, and student readiness for m-learning. Other learner-focused papers include those on attrition and retention, group work, and learners' intentions. These papers are followed by a diverse mix of papers investigating applications, quality, videos, MOOCs, and implementations.

The first paper by Chang, Chiu, and Huang investigated the use of mind-mapping to help sixth-grade (K-12) students to learn using mobile devices. The investigators added digital archive data to encourage students to collaborate to develop their knowledge, achieving positive results.

Kocdar, Karadeniz, Bozkurt, and Buyuk developed a scale for determining the self-regulated learning skills of distance learners in self-paced open and distance learning courses. Their analysis suggested that goal-setting, help-seeking, self-study strategies, managing physical environment, and effort regulation, were important in promoting self-pacing and in helping students to acquire new skills in an online environment.

In their investigation of students participating in connectivist learning, Wang, Anderson, and Chen tracked a cMOOC. They analysed how blogs, Twitter, Facebook, and video-conferencing supported their learning and social interactions. Results suggest that learners could be divided into four categories: unconnected floaters, connected lurkers, connected participants, and active contributors.

Costley and Lange investigated motivation and its relationship to cognitive load in semi-formal group work settings. This supports previous research showing that groups benefit low-motivated students more than the highly motivated.

Virtual reality is the subject of Huang and Liaw's paper in which they take a constructivist approach. They attempt to establish effective techniques to motivate learning among students. Their research demonstrates that the perception of self-efficacy and interactions are important indicators of learning motivation to use VR.

The discourse on Twitter surrounding openness was investigated by Paskevicius, Veletsianos, and Kimmons. Using a mixed-method analysis, they followed the development of the openness conversation from 
OER to include emerging discussion on open practices.

The status of quality in a Kenyan university is the subject of Hadullo, Oboko, and Omwenga. This investigation includes quality issues relating to course design, content support, social support, administrative support, course assessment, learner characteristics, instructor characteristics, and institutional factors.

Using the Technology Acceptance Model (TAM), Nagy looked at video usage in a Mathematics course. Learning performance and interactions were also examined. Results showed that learner perceptions had a direct effect on video usage, and this usage had a significant effect on both learning performance and learner satisfaction.

In another video study, Bayeck and Choi looked at cultural dimensions in MOOCs' introductory videos. The authors found that the videos reflect the culture of the countries that created them and offer suggestions for creating such introductory videos.

Adham, Parslow, Dimitiadi, and Lundqvist studied segregated gender in MOOCs using avatars. The authors created a female avatar as a tutor for social interactions in order to address concerns raised by gender segregation.

Zawacki-Richter, Bozkurt, Alturki, and Aldraiweesh conduct a content analysis of research on MOOCs. They reviewed hundreds of articles using a test-mining tool. They found that research focuses on four main lines of research including challenges, platforms, learners/content, and quality.

Examining MOOCs in Tourism and Hospitality, Lin and Cantonie explored the experiences of instructors as they decided to implement and deliver the MOOCs. Findings show that the institution's interest in MOOCs was the prime motivator. The different phases are described including the instructors' proclivity for risk avoidance, but who nevertheless continued to participate in MOOC delivery.

Chorianopoulos has created a taxonomy of educational video formats that attempts to allow for comparison studies and meta-analyses. The taxonomy establishes a comprehensive design space for understanding the learning video landscape.

The final paper returns us to the K-12 sector with Taylor and McNair's paper describing virtual school startups in California. Interviews found the following as critical for the success of the startups: establishing founder, preliminary research, district support, teacher and staff selection, financial evaluation, and curriculum decisions.

A Research Note and a book review round out this issue. In Turkey, Ozcan examined the motives of administrators for establishing online programs and Cifuentes gives us a book review of an elearning handbook.

\section{Athabasca University}

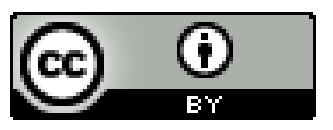

